20

\title{
Дифференциация пигментных новообразований кожи на основе цифровой обработки оптических изображений*
}

\author{
(c) Е.Н. Римская ${ }^{1}$, А.О. Щадько ${ }^{1}$, И.А. Аполлонова ${ }^{1}$, А.П. Николаев ${ }^{1}$, А.Н. Брико ${ }^{1}$, И.А. Дешин ${ }^{1}$, \\ П.Ю. Бережной ${ }^{2}$, К.Г. Кудрин ${ }^{3}$, К.И. Зайцев ${ }^{1,4,5}$, В.В. Тучин ${ }^{6}$, И.В. Решетов ${ }^{4}$ \\ ${ }^{1}$ Московский государственный технический университет им. Н.Э. Баумана, \\ 105005 Москва, Россия \\ ${ }^{2}$ Российский экономический университет им. Г.В. Плеханова, \\ 117997 Москва, Россия \\ ${ }^{3}$ Институт повышения квалификации Федерального медико-биологического агентства России, \\ 125371 Москва, Россия \\ ${ }^{4}$ Первый Московский государственный медицинский университет им. И.М. Сеченова, \\ 119991 Москва, Россия \\ ${ }^{5}$ Институт общей фозики им. А.М. Прохорова Российской Академии Наук, \\ 119991 Москва, Россия \\ ${ }^{6}$ Саратовский государственный университет им. Н.Г. Чернышевского, \\ 410012 Саратов, Россия \\ e-mail: romehelen@gmail.com, reshetoviv@mail.ru
}

Поступила в редакцию 21.11.2018 г.

В окончательной редакции 09.01.2019 г.

Принята к публикации 31.01.2019 г.

\begin{abstract}
Предложен метод дифференциации пигментных новообразований кожи на основе цифровой обработки оптических изображений. Регистрация изображений осуществляется цифровой фотокамерой с использованием эталона для ее цветовой и пространственной калибровки. Метод предполагает сегментацию и автоматическое распознавание новообразований на основе 5 параметров - диаметр, площадь, цвет, форма и резкость границы. В результате анализа выявляются клинические признаки новообразования, необходимые для дифференциальной диагностики, и рассчитывается вероятность его малигнизации. Проведена апробация метода на 360 цифровых изображениях пигментных новообразованиях кожи in vivo, включая обыкновенные, диспластические невусы и меланому кожи. Чувствительность и специфичность метода составили 97 и $95 \%$ соответственно.
\end{abstract}

DOI: $10.21883 /$ OS.2019.05.47657.6-19

\section{Введение}

Меланома занимает особое положение среди других новообразований кожи из-за своей чрезвычайной агрессивности $[1,2]$. На сегодняшний день она остается главной причиной смерти больных в онкодерматологии, причем за последние два десятилетия частота заболеваемости меланомой резко возросла $[1,3,4]$. Среднегодовой темп прироста стандартизированных показателей заболеваемости меланомой кожи составляет от 3 до 7\% [5-8]. Лечение новообразований кожи наиболее эффективно на 1-й и 2-й стадиях ее развития (пятилетняя выживаемость составляет более $80 \%$ ) по сравнению с 3-й и 4-й стадиями (пятилетняя выживаемость - не более 20\%) [2-7]. Нередко больной обращается к врачу, когда опухоль имеет распространенный характер (глубокую инвазию и метастазы), либо длительное время наблюдается у дерматологов, косметологов, хирургов

\footnotetext{
* The 22nd Annual Conference Saratov Fall Meeting 2018 (SFM'18): VI International Symposium „Optics and Biophotonics“ and XXII International School for Junior Scientists and Students on Optics, Laser Physics \& Biophotonics, September 24-29, 2018, Saratov, Russia. https://www.sgu.ru/structure/fiz/saratov-fall-meeting/previousconferences/saratov-fall-meeting-2018
}

с ошибочным диагнозом и соответственно получает ошибочное лечение, что может стимулировать развитие злокачественного новообразования $[8,9]$.

Самым распространенным методом диагностики злокачественных новообразований кожи является морфологическое исследование удаленных биотканей - гистологическое исследование [2-6]. Однако иссечение новообразования проводят главным образом в случае выраженных клинических признаков его злокачественности, поэтому необходима ранняя дифференциальная диагностика, с которой без современных инструментов способны справиться только высококвалифицированные медицинские работники [3,7]. Таким образом, разработка новых методов ранней диагностики злокачественных новообразований кожи является актуальной проблемой современной медицины, онкологии, прикладной физики и инженерных наук [1-7].

На современном этапе в дерматологии при постановке диагноза распространен визуальный осмотр [3-10], в то время как результаты других исследований (дерматоскопия [10-14], конфокальная лазерная сканирующая микроскопия [15-17], термометрия [18-20], высокочастотное ультразвуковое сканирование кожи [21,22], флу- 
оресцентная диагностика [23,24], кроссполяризационная оптическая когерентная томография [25,26], спектроскопия и визуализация в терагерцовом диапазоне [27,28]) имеют уточняющий характер [9-17]. Перечисленные методы неинвазивной диагностики пигментных новообразований кожи обладают как достоинствами, так и недостатками. Чувствительность и специфичность (соответственно 92.4 и 93.7\%) дерматоскопии [10-14] для диагностики пигментных новообразований кожи очень высоки, однако этот метод пока имеет ограниченное применение в России. В России до сих пор распространена визуальная оценка каждого новообразования [3-10]. Эффективность такой диагностики определяется опытом врача (специфичность и чувствительность врача общего профиля составляет от 30 до 50\%, для эксперта - около 90\%), поэтому при постановке диагноза высока вероятность ошибки субъективного характера [29,30]. Флуоресцентная диагностика (чувствительность - 87.2\% и специфичность - 94.8\%) не позволяет четко проводить дифференциальную диагностику злокачественных новообразований и различных фоновых и предраковых заболеваний кожи $[23,24]$. Совпадение термометрического и гистологического диагнозов имеет место в 94.8\% случаев при меланоме кожи и в $67.9 \%$ при доброкачественных новообразованиях кожи. Точность термометрического метода ограничивается тем обстоятельством, что далеко не все меланомы кожи обладают свойствами гипертермии, что не позволяет проводить дифференциальную диагностику меланомы кожи [18-20]. Высокочастотное ультразвуковое сканирование кожи не позволяет отличить меланому кожи от меланоцитарного невуса из-за схожести эхографических признаков доброкачественных и злокачественных новообразований [21,22]. Исследования по применению методов конфокальной лазерной микроскопии и кроссполяризационной оптической когерентной томографии стали появляться сравнительно недавно и имеющиеся результаты пока не позволяют говорить об их диагностической эффективности по сравнению с другими методами [15-17,25,26].

Несмотря на значительный прогресс в развитии инструментальных средств диагностики, по-прежнему не удается переломить тенденцию ежегодного роста заболеваемости населения злокачественными новообразованиями кожи.

Цифровая обработка оптических изображений представляется перспективным путем решения проблемы диагностики новообразований кожи. Значительное развитие элементной базы оптической визуализации, достаточно низкая стоимость современных систем регистрации изображений, возможность их обработки в реальном масштабе времени (например, с векторизацией вычислений на графических картах), наличие систем визуализации на большинстве мобильных платформ (мобильные телефоны и планшеты) делают создание новых методов диагностики новообразований кожи на основе цифрового имиджинга крайне привлекательным [31-33].
В то же время создание новых методов и устройств требует решения ряда проблем, среди которых стоит особо отметить необходимость оптимизации алгоритмов дифференциации новообразований для обеспечения максимальной чувствительности и специфичности диагностики, а также апробации новых методов на фантомах и образцах тканей ex vivo и in vivo.

В настоящей работе предлагается метод дифференциации пигментных новообразований кожи на основе цифровой обработки их оптических изображений, с целью раннего выявления меланомы кожи. Областью применения данного метода служит сфера медицины (в частности, дерматология и онкология). Метод предполагает использование эталона - пластиковой карты с палитрой цветовой системы RGB (R - red; G green; B - blue) и шкалой размеров. Изображение эталона регистрируется камерой одновременно с новообразованием, что позволяет скорректировать передачу цветовых характеристик новообразования в различных условиях съемки и определить его истинный размер. Метод включает следующие основные этапы работы: регистрацию изображения новообразования и эталона, размещенного на поверхности кожи; оценку качества изображений; распознавание и обработку эталона; распознавание пигментного новообразования; определение параметров новообразования - диаметр, площадь, цвет, форма, резкость границ; вычисление вероятности малигнизации. Последний параметр используется для дифференциации новообразований на доброкачественные и злокачественные. Оригинальный подход к сегментации и распознаванию новообразований позволяет с высокой точностью определить его границы, что является ключевым фактором для дальнейшего анализа его параметров $[4,34,35]$. Разработанный классификатор цвета позволяет определять один из восьми цветов, используемых во врачебной практике, при любых условиях освещения. Форма пигментных новообразований классифицируется на три группы: круглая, овальная и произвольная. Расчет вероятности малигнизации позволяет оценить степень риска перерождения новообразование в злокачественное. Для экспериментального исследования были отобраны 3 тестовые группы изображений пигментных новообразований кожи in vivo с подтвержденным диагнозом. В результате исследования изображений 360 пигментных новообразований кожи (доброкачественные новообразования - 165 изображений, группа риска 185 изображений и злокачественные новообразования 10 изображений) установлена высокая эффективность их дифференциации с помощью предложенного метода. Чувствительность и специфичность дифференциации составили 97 и 95\%, что существенно превосходит аналогичные показатели для врача общего профиля и эксперта $[3,10]$. К достоинствам предложенного метода дифференциации пигментных новообразований кожи можно отнести неинвазивность, возможность автоматического определения параметров новообразований, а также обнаружение предраковых изменений на их основе. 


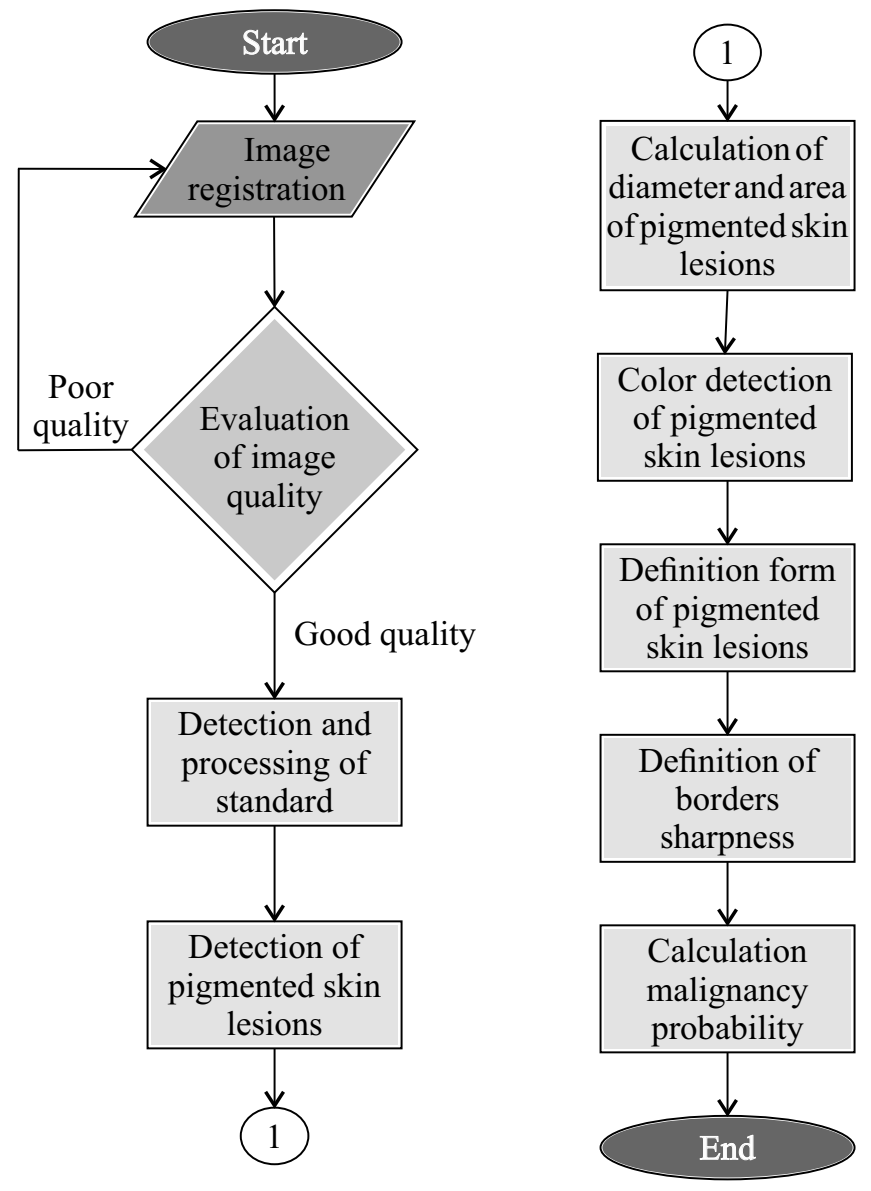

Рис. 1. Алгоритм метода диагностики пигментных новообразований кожи на основе цифровой обработки оптических изображений.

\section{1. Метод дифференциации пигментных новообразований}

Метод дифференциации пигментных новообразований кожи включает регистрацию изображений новообразования и эталона, его последующую цифровую обработку и анализ [36-38]. Регистрация изображений осуществляется цифровой фотокамерой, после чего оцениваются параметры качества изображения - яркость, контрастность, тоновая насыщенность и резкость. Если качество изображения приемлемо, то алгоритм переходит к следующему этапу обработки, включающему нахождение элементов эталона и его параметров - значений цветов RGB и площади пикселя на изображении. Далее происходит обнаружение и распознавание самого пигментного новообразования и определение его параметров диаметр, площадь, цвет, форма и резкость границы. На основе перечисленных параметров вычисляется вероятность малигнизации новообразования [34,35]. Алгоритм дифференциации пигментных новообразований кожи по предложенному методу представлен на рис. 1 .

\section{1. Регистрация изображения пигментного новообразования}

Значительная вариабельность геометрии и цветовых параметров новообразований позволяют сформулировать требования к системе регистрации их цифровых оптических изображений. Размеры новообразования в зависимости от его морфологии могут составлять от нескольких долей миллиметров до целых анатомических областей (врожденный невоклеточный невус). Из соображений сокращения времени обследования и с учетом характерных анатомических размеров человека целесообразно выбрать размер поля зрения в плоскости объекта исследования $\sim 50 \times 50 \mathrm{~mm}^{2}$. В отсутствие данных о спектре изрезанности границ новообразования при определении необходимого разрешения системы следует задаться размером минимального разрешаемого объекта. Основываясь на описании вторичных диагностических признаков меланомы, в качестве такого объекта можно принять придатки кожи. Например, характерная толщина среднего волоса составляет $80-110 \mu \mathrm{m}$, а размер потовой железы 30-35 $\mu \mathrm{m}$ [39].

Отмеченные условия могут быть обеспечены множеством современных цифровых фотоаппаратов. В частности, в данной работе для экспериментальных исследований была выбрана цифровая фотокамера Canon EOS 550D со следующими характеристиками:

- максимальное разрешение $5184 \times 3456$;

- размер матрицы $22.3 \times 14.9 \mathrm{~mm}$, а также объектив Canon EFS $18.55 \mathrm{~mm}$ со следующими характеристиками:

- фокусное расстояние $18-55 \mathrm{~mm}$;

- диафрагма $(\mathrm{max} / \mathrm{min})(1: 3.5-1: 5.6) /(1: 22-1: 38)$.

Используя формулы геометрической оптики, сделаем пересчет поля зрения и разрешения из плоскости матрицы в плоскость объекта (разрешение по дифракционному пределу) (табл. 1). Дифракционный предел разрешения в плоскости объекта равен $0.013 \mathrm{~mm}$, а поле зрения в плоскости объекта равно $151 \mathrm{~mm}$, что удовлетворяет заданным параметрам.

Регистрация изображений пигментных новообразований кожи проводилась по следующей схеме: к участку тела рядом с новообразованием прикладывался эталон (разд. 1.2), затем данное новообразование вместе с эталоном фотографируется фотокамерой перпендикулярно эталону на расстоянии приблизительно равном $10 \mathrm{~cm}$ (рис. 2,a). Источником света служит встроенная в фотокамеру вспышка. Данные с фотокамеры передаются на персональный компьютер, и затем производится анализ и обработка цифровых изображений пигментных новообразований кожи.

Используемая при регистрации изображений фотовспышка на расстоянии $10 \mathrm{~cm}$ обеспечивает достаточную однородность светового потока, не зависящую от освещения в помещении. Для максимальных новообразований (диаметром $3 \mathrm{~cm}$ ) неоднородность освещения составляет менее $5 \%$.

За приведение всех изображений к единой цветопередаче и устранение ошибок, связанных с отклонением от 

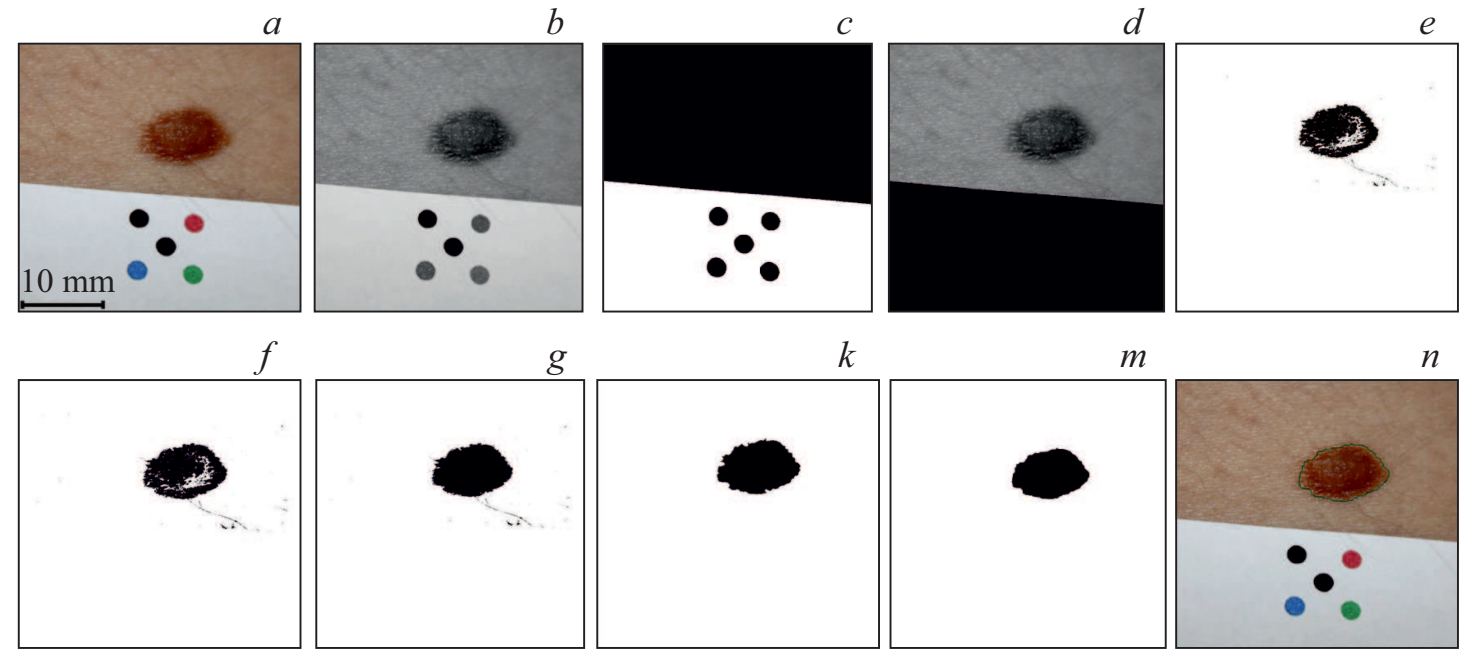

Рис. 2. Метод детектирования границ новообразования: $a$ - исходное RGB-изображение; $b$ - преобразование RGB-изображения в полутоновое; $c$ - распознавание и обработка эталона; $d-$ удаление эталона с изображения; $e-$ выделение границ методом выращивания областей (создание бинарной градиентной маски); $f$ - наращение бинарного изображения (дилатация); $g$ заполнение отверстий исследуемого объекта на изображении; $k$ - удаление лишних объектов на изображении (например, волоса); $m$ - сглаживание границ объекта методом утончения границ (эрозии); $n$ - выделение границы.
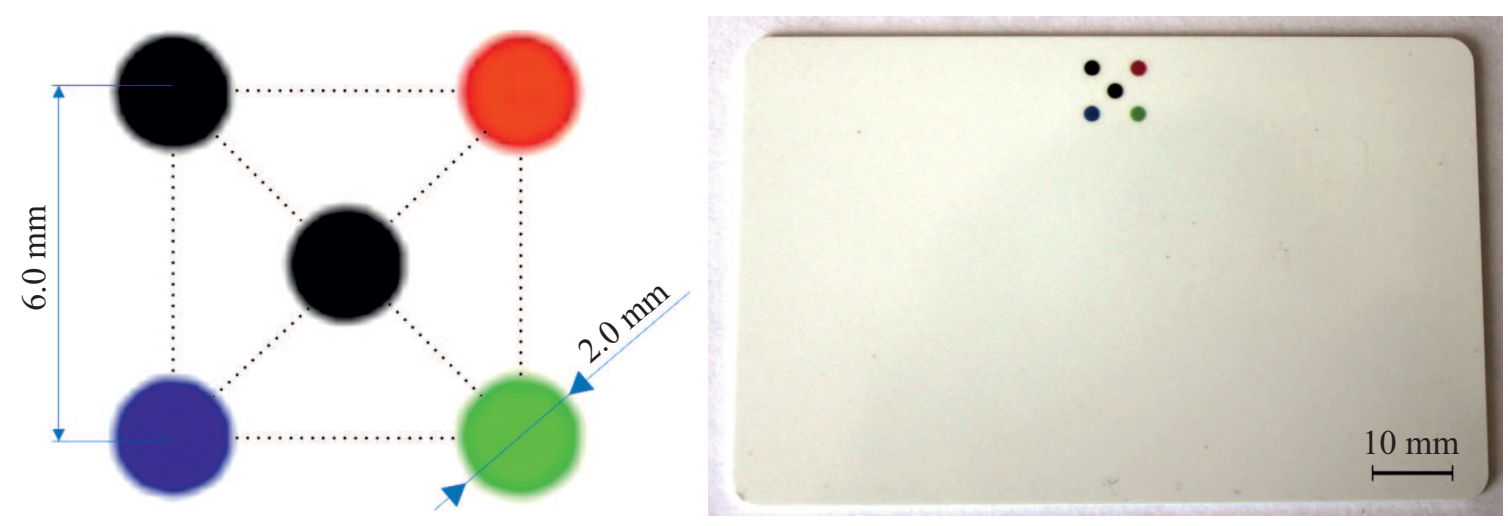

Рис. 3. Эталон для регистрации изображений: $a-$ модель эталона, $b-$ фотография напечатанного эталона.

перпендикулярности, отвечает калибровка и коррекция пространственных искажений по эталону (разд. 1.2).

\section{2. Распознавание и обработка эталона}

Эталон - пластиковая карта с цветовой палитрой RGB. Его применяют для корректной передачи цветовых характеристик пигментных новообразований кожи (цвет) и шкалу размеров (диаметры кругов равны $2 \mathrm{~mm}$ и расположены на одинаковом расстоянии друг от друга) для определения истинных размеров пигментных новообразований кожи (диаметр, площадь). Выбор цветов эталона обусловлен особенностями выходного цветового пространства цифровой фотокамеры (цветовая модель RGB).

Эталон имеет простой вид, что удобно для быстрого и правильного его нахождения на изображениях при сегментации (рис. 3). По результатам калибровки цвета по эталону вычисляется вектор цветовой ошибки и по нему устраняется ошибка для каждого пикселя (вычитается). Согласно ISO 12647-2:2004, погрешность цвета при офсетной печати составляет менее $2.5 \%$.

Также эталон служит для устранения ошибок, связанных с отклонением от перпендикулярности. Геометрические отклонения, обусловленные неперпендикулярностью оптической оси фотоаппарата к предметной плоскости, составляют $\pm 15 \%$. Эти искажения, вносимые неперпендикулярностью легко устраняются с помощью аффинных преобразований [40].

Для нахождения 5 кругов использовался метод сегментации, основанный на операторе выделение границ Канни $[34,35]$ (рис. 2,c-d). По сегментированным областям кругов определялись $\mathrm{R}, \mathrm{G}, \mathrm{B}$,составляющие каждой из 5 областей для корректной передачи цветовых характеристик пигментного новообразования кожи, а также для определения масштаба изображения (рассчитывалось цифровое разрешение в $\mathrm{pixel} / \mathrm{mm}$ ). 
Таблица 1. Параметры оптической системы

\begin{tabular}{|c|c|c|c|c|c|c|}
\hline \multicolumn{2}{|c|}{ Параметры объектива } & \multirow{2}{*}{$\begin{array}{c}\text { Поле } \\
\text { зрения }\end{array}$} & \multirow{2}{*}{\begin{tabular}{|} 
Расстояние \\
от объектива \\
до объекта $p$
\end{tabular}} & \multirow{2}{*}{$\begin{array}{c}\text { Расстояние } \\
\text { от объектива } \\
\text { до изображения } \\
\text { объекта на матрице, } p^{\prime} \\
\frac{1}{p}+\frac{1}{p^{\prime}}=\frac{1}{f}\end{array}$} & \multirow{2}{*}{$\begin{array}{c}\text { Дифракционный предел } \\
\text { разрешения на матрице, } \\
r^{\prime}=1.22 \cdot \lambda \cdot \frac{p^{\prime}}{D}\end{array}$} & \multirow{2}{*}{$\begin{array}{c}\text { Дифракционный предел } \\
\text { разрешения на объекте, } r \\
\frac{r^{\prime}}{p^{\prime}}=\frac{r}{p}\end{array}$} \\
\hline $\begin{array}{c}\text { Фокусное } \\
\text { расстояние } \\
f_{\min }\end{array}$ & \begin{tabular}{|c} 
Диаметр \\
входного \\
зрачка $D_{\min }$
\end{tabular} & & & & & \\
\hline & $5.14 \mathrm{~mm}$ & $151 \mathrm{~mm}$ & $100 \mathrm{~mm}$ & $21.95 \mathrm{~mm}$ & $2.9 \cdot 10^{-6} \mathrm{~m}$ & $13.21 \cdot 10^{-6} \mathrm{~m}$ \\
\hline
\end{tabular}

Таблица 2. Параметры нахождения значения малигнизации

\begin{tabular}{|c|c|c|c|}
\hline \multirow{2}{*}{ Параметр } & \multicolumn{3}{|c|}{ Оценка } \\
\hline & Значение & Баллы & Весовые коэффициенты \\
\hline Форма, $F$ & $\begin{array}{l}0-\text { круглая } \\
1-\text { овальная } \\
2-\text { произвольная }\end{array}$ & $0-2$ & 0.1 \\
\hline Резкость границы, $R$ & $\begin{array}{l}0-\text { резкая } \\
1-\text { нерезкая }\end{array}$ & $0-1$ & 0.008 \\
\hline Цвет родинки, $C$ & $\begin{array}{l}1 \text { - светло-коричневый } \\
2 \text { - коричневый } \\
3 \text { - темно-коричневый } \\
4 \text { - желто-коричневый } \\
5 \text { - серый } \\
6 \text { - голубоватый } \\
7 \text { - розово-пурпурный } \\
8 \text { - черный }\end{array}$ & $1-8$ & 0.04 \\
\hline $\begin{array}{c}\text { Максимальный диаметр, } \\
\qquad, \mathrm{mm}\end{array}$ & $\begin{array}{l}1-<2 \\
2-2.01-4 \\
3-4.01-6 \\
4-6.01-8 \\
5-8.01-10 \\
6->10\end{array}$ & $1-6$ & 0.04 \\
\hline Площадь, $S, \mathrm{~mm}^{2}$ & $\begin{array}{l}1-<3.14 \\
2-3.15-12.56 \\
3-12.57-28.26 \\
4-28.27-50.24 \\
5-50.25-78.5 \\
6->78.5\end{array}$ & $1-6$ & 0.04 \\
\hline
\end{tabular}

\section{3. Распознавание пигментного новообразования кожи}

Для обнаружения пигментного новообразования кожи использовались следующие методы (рис. $2, a-n)$ :

1) Метод выращивания областей. Метод представляет собой процедуру, которая группирует пикселы или подобласти в более крупные области по заранее заданным критериям роста. Основной подход состоит в том, что на исходном изображении выбираются точки, играющие роль .центров кристаллизации. (точки с максимальным уровнем яркости), а зачем на них наращиваются области путем добавления к каждому центру тех соседних пикселов, которые по своим свойствам близки к центру кристаллизации (используется определенный критерий их близости - разница в яркости) [34,35,38]. Значение яркости пикселов, которые являются центрами кристаллизации, равно 55, а значение глобального порога, который применяется для проверки соседних (8-связных) пикселов для центров кристаллизации на предмет того, достаточно ли они „Похожи“ на них, равно 40. Данные значения были получены опытным путем при анализе верифицированной базы данных изображений пигментных новообразований кожи. В результате получим бинарную градиентную маску, отображающую линии с высоким контрастом на изображении (рис. 2,e).

2) Метод уточнения границ. Данный метод представляет собой уточнение уже найденных ранее границ би- 


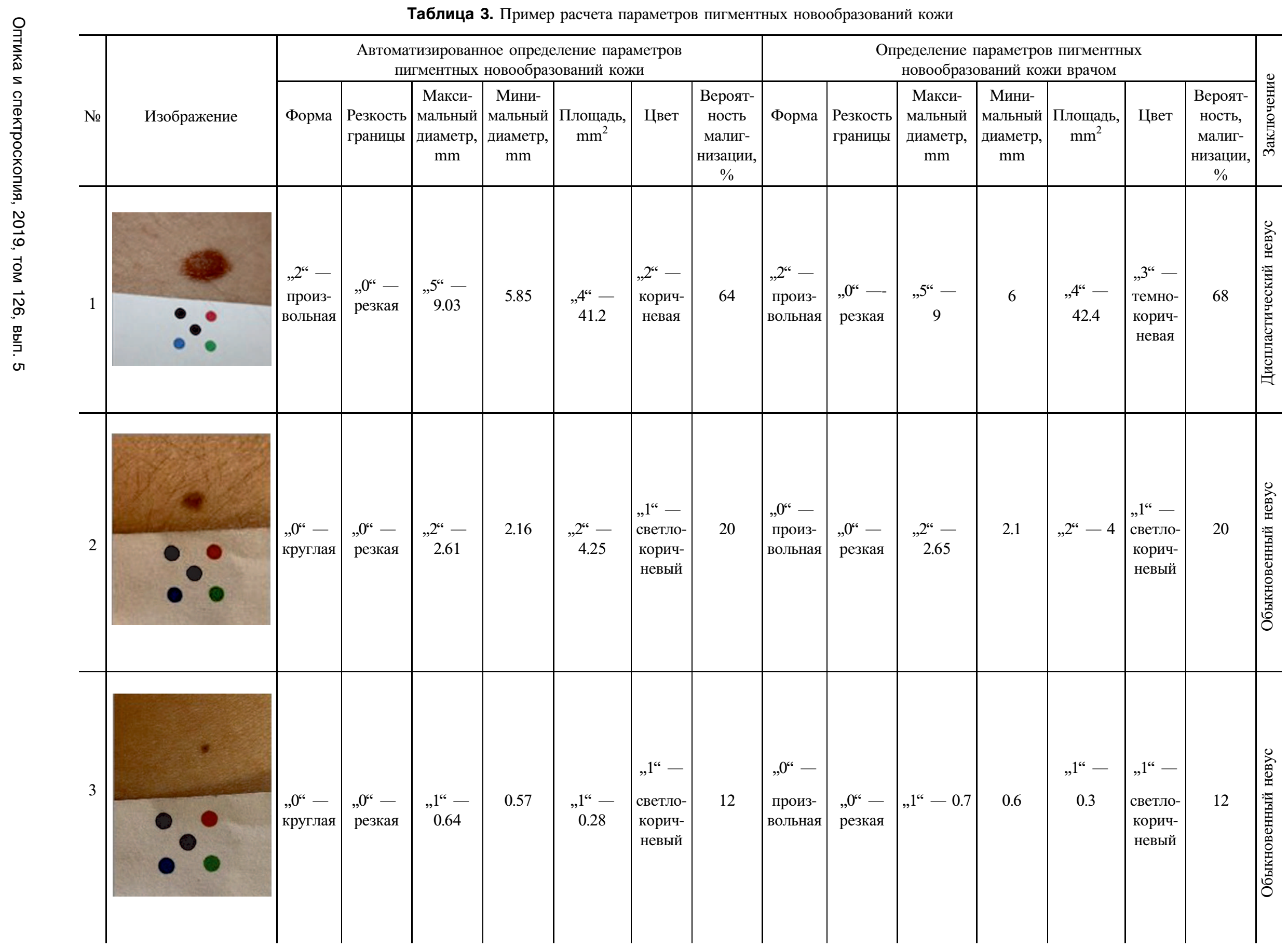


Таблица 3. Продолжение.

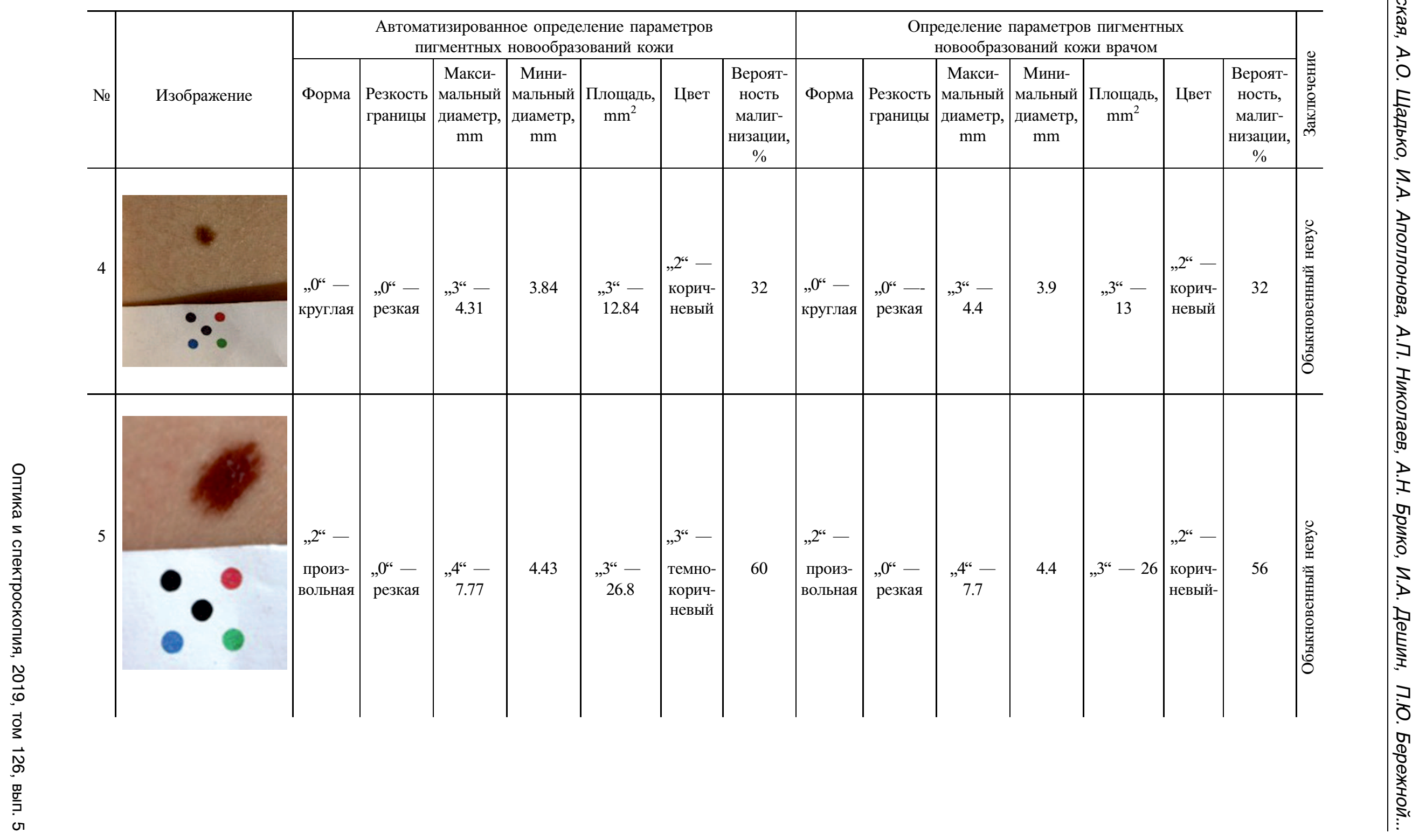




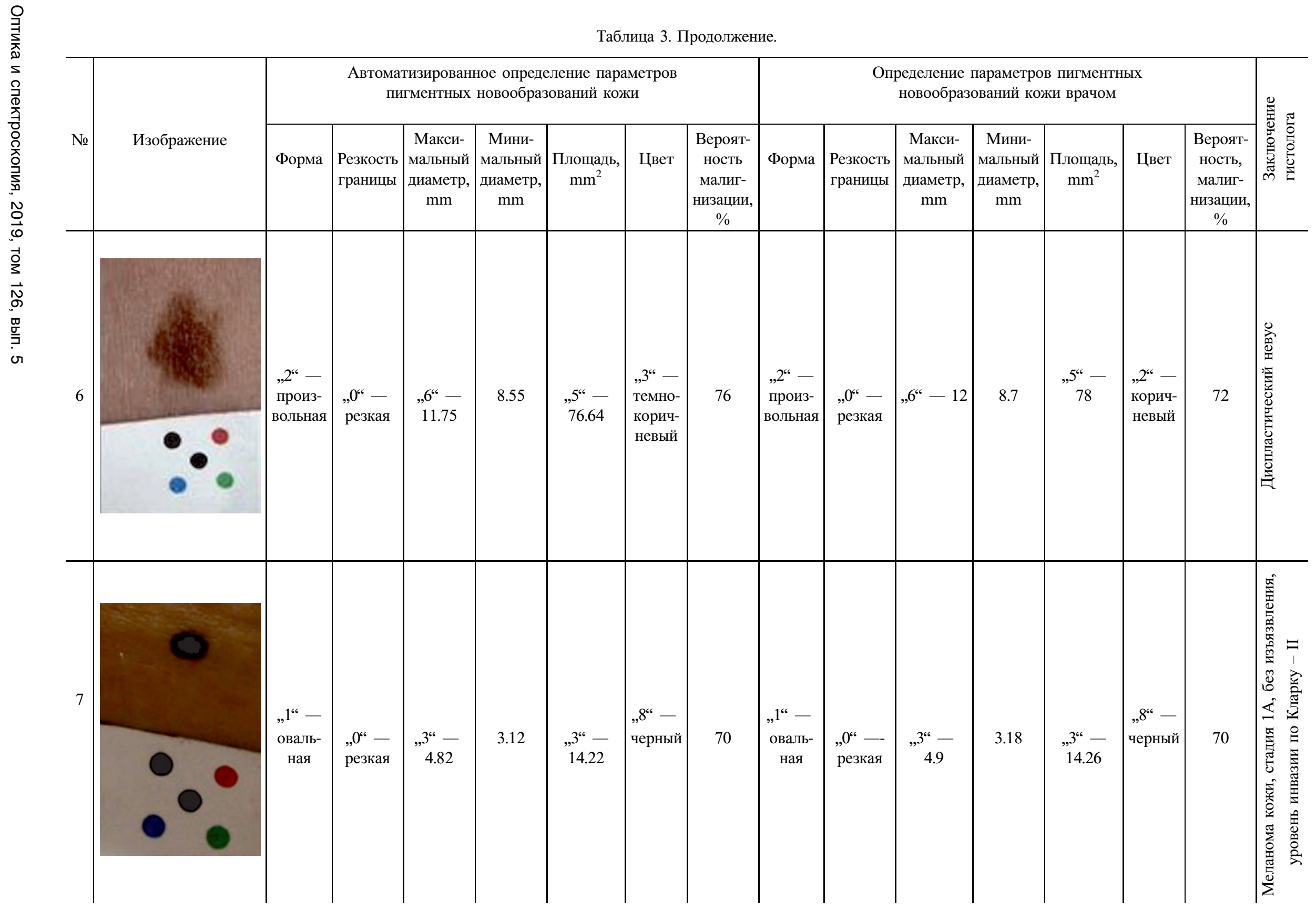


Таблица 3. Продолжение.

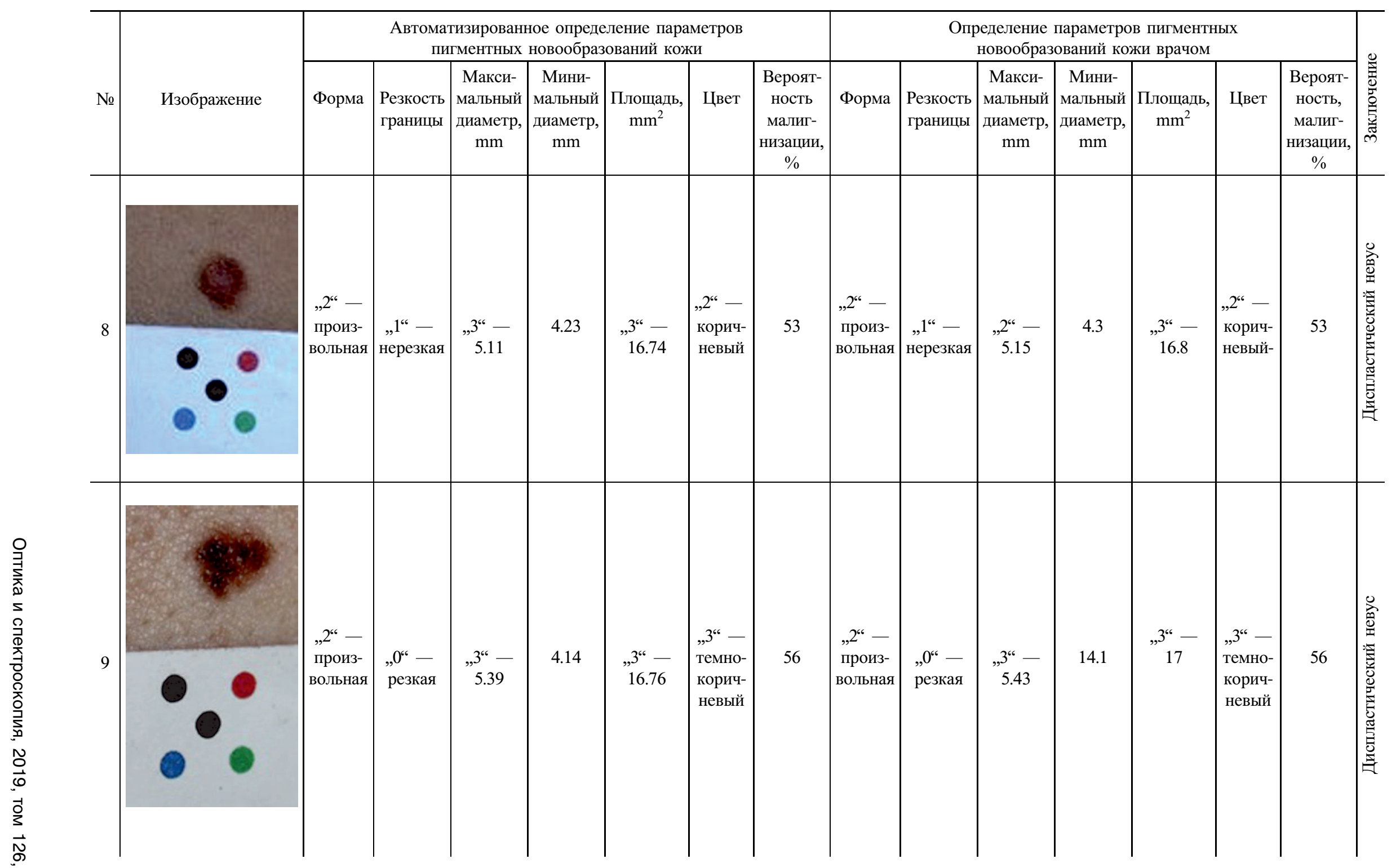


нарной градиентной маски. С помощью функции морфологического расширения границ (дилатации) (рис. 2,f) был получен неразрывный контур новообразования для того, чтобы впоследствии при устранении лишних объектов не потерять полезную информацию. Для получения конечного контура искомого новообразования без посторонних включений проводилось заполнение внутренних промежутков объекта (рис. 2,g). Далее происходит очистка от пограничных объектов (например, волос) (рис. 2,k). Происходит „гашение“ структур, которые ярче своих окрестностей и которые примыкают к границам изображения. Данная операция не изменяет границу новообразования. Далее происходит сглаживание границ объекта методом утончения границ (эрозии) (рис. 2, m) [34,35,38].

В результате данных процедур была получена граница пигментного образования кожи (рис. $2, n)$.

\section{4. Определение диаметра и площади пигментного новообразования кожи}

После нахождения границы пигментного новообразования кожи, исходя из размеров 1 пикселя, были рассчитаны диаметр и площадь пигментного новообразования кожи.

\section{5. Определение цвета пигментного новообразования кожи}

При диагностике меланомы кожи врачи выделяют восемь цветов пигментных новообразований: светло-коричневый, коричневый, темно-коричневый, желто-коричневый, серый, голубоватый, розово-пурпурный и черный $[4,9,12]$.

Для распознавания цвета был разработан универсальный классификатор, содержащий все возможные цвета новообразований, зарегистрированные при различных условиях освещения. При использовании данного классификатора цвет новообразования на изображении определялся как принадлежащий к одному из выше указанных восьми цветов.

\section{6. Определение формы пигментного новообразования кожи}

Пигментные новообразования различаются не только по цветовым характеристикам между собой, но также по форме и размерам [38-42]. В норме пигментное новообразование должно быть круглой или эллипсоидной формы, при перерождении пигментного новообразования в злокачественную опухоль форма становится произвольной [1-3,9,38-42]. Для нахождения формы пигментного новообразования определялись следующие морфомерические признаки: площадь выпуклого многоугольника, эксцентриситет и коэффициент заполнения [32,34,35,43-47].

Для расчета произвольной формы вычислялись:
1) разница между площадью новообразования и площадью выпуклого многоугольника, в который вписано новообразование.

2) коэффициент заполнения (равен отношению площади объекта к площади ограничивающего прямоугольника).

По результатам анализа верифицированной базы данных изображений пигментных новообразований кожи данная разница между площадями превышает значение в 5000. Данная разница для новообразований круглой и эллипсоидной формы намного меньше. А коэффициент заполнения находится в диапазоне между 0.600-0.720.

Для нахождения круглой и овальной форм рассчитывались эксцентриситет и коэффициент заполнения. Для круглой формы эксцентриситет менее 0.600, а коэффициент заполнения более 0.780 .

В результате новообразование классифицировалось на 3 группы: круглая форма, овальная форма и произвольная форма.

\section{7. Определение резкости границы пигментного новообразования кожи}

В норме пигментное новообразование должно иметь резкую границу, размытие границы новообразования говорит о начале перерождения в меланому кожи [1,2-6,9,38--44].

Резкость границы находилась по первой производной интерполированной кривой интенсивности пикселей полутонового изображения на границе новообразования [37]. По экспериментальным данным был найден нормированный максимальный пограничный градиент интенсивности (максимальный угол наклона кривой), равный 0.3. В результате чего граница новообразования классифицировалась на резкую (градиент интенсивности меньше 0.3) и нерезкую (градиент интенсивности больше 0.3$)$.

\section{8. Определение вероятности малигнизации пигментного новообразования кожи}

При нахождении вероятности малигнизации врачи опираются на алгоритм ABCD [41-44], при котором каждому клиническому признаку (форма $-F$, резкость границы $-R$, цвет $-C$, площадь $-S$ и диаметр $D$ пигментных новообразований кожи) дается оценка в баллах и коэффициент этого признака $\left(k_{F}, k_{R}, k_{C}\right.$, $\left.k_{D}, k_{S}\right)$. Ниже приведена таблица алгоритма нахождения вероятности малигнизации по расчетным параметрам (табл. 2).

Данный алгоритм позволяет дифференцировать доброкачественный и злокачественный характер пигментных новообразований кожи на основании анализа найденных выше параметров:

Значение вероятности малигнизации Р находится:

$$
P=\left(k_{F} F+k_{R} R+k_{C} C+k_{D} D+k_{S} S\right) \cdot 100 \%,
$$

где $k_{F}, k_{R}, k_{C}, k_{D}, k_{S}$ - безразмерные коэффициенты, учитывающие вклад параметров новообразований. В хо- 
де исследований 360 пигментных новообразований кожи экспериментально установлено, что для доброкачественных новообразований вероятность малигнизации находится в промежутке от $12 \%$ до $42 \%$, для новообразований из группы риска - от $43 \%$ до $67 \%$, а для злокачественных новообразований - от $68 \%$ до $100 \%$.

\section{2. Апробация на тестовой выборке}

Программная реализация данного метода диагностики осуществлялась на языке программирования MATLAB (рис. 2, $a-n)$. Пример результата расчета параметров пигментных новообразований кожи представлен в табл. 3.

Для экспериментального исследования были отобраны 3 тестовые группы изображений пигментных новообразований кожи с подтвержденным диагнозом: группа с изображениями доброкачественных новообразований (165 изображений), группа риска (185 изображений) и злокачественные новообразования (10 изображений). Данная верифицированная база изображений пигментных новообразований кожи собиралась в НОКЦ пластической хирургии Первого МГМУ им. И.М. Сеченова и Государственном научном центре дерматовенерологии и косметологии.

По данным исследования диагностическая чувствительность метода составила 97\%, специфичность 95\%. По результатам исследования установлена высокая диагностическая эффективность метода (чувствительность и специфичность > 90\%).

Предложенный метод диагностики пигментных новообразований кожи на основе цифровой обработки оптических изображений может быть использован в различных областях науки и техники, где необходима обработка цифровых изображений для дифференциальной диагностики. Наряду с отмеченным данный метод позволяет обнаруживать патологию на ранней стадии, что существенно облегчает лечение больных, а значит, имеет высокий социально-экономический эффект.

\section{Заключение}

По результатам проведенных исследований установлена возможность дифференциации пигментных новообразований кожи при использовании цифровой обработки оптических изображений. Для реализации данного метода дифференциации был разработан алгоритм анализа оптических изображений пигментных новообразований кожи, который определяет параметры новообразований (диаметр, площадь, форма, цвет и резкость границы) и рассчитывает вероятность малигнизации для обнаружения патологии на ранней стадии. Были сформулированы требования к начальным условиям регистрации изображений, а также разработан алгоритм цветокалибровки получаемых изображений при помощи эталона. С целью повышения точности нахождения контура пигментных новообразований кожи была использована двойная сегментация изображений (сегментация методом выращивания областей и методом уточнения границ). Чувствительность данного метода составила 97\%, специфичность - 95\%.

В дальнейшем планируется разработка программного обеспечения для экспресс-диагностики пигментных новообразований кожи с целью раннего выявления меланомы кожи, сравнение параметров новообразований с течением времени (временная динамика).

\section{Финансирование работы}

Создание экспериментальной установки, обработка и анализ изображений пигментных новообразований кожи поддержаны Российским фондом фундаментальных исследований (РФФИ), проект № 18-38-00958. Экспериментальные исследования выполнены при поддержке РФФИ, проект № 18-29-02099.

\section{Соблюдение этических норм}

Все исследования и процедуры с участием людей соответствуют этическим стандартам институционального и/или национального комитета по исследовательской этике и Хельсинкской декларации 1964 года и ее последующим изменениям или сопоставимым нормам этики. От каждого из включенных в исследование участников было получено информированное добровольное согласие.

\section{Конфликт интересов}

Авторы заявляют, что у них нет конфликта интересов.

\section{Список литературы}

[1] Eisemann N., Waldmann A., Geller A.C., Weinstock M.A., Volkmer B., Greinert R., Breitbart E.W., Katalinic A. // J. Invest. Dermatol. 2014. V. 134. N 1. P. 43-50. doi 10.1038/jid.2013.304

[2] LeBoit P.E., Burg G., Weedon D., Sarasain A. World Health Organization Classification of Tumours. Pathology and Genetics of Skin Tumours. Lyon: IARC Press, 2006. 355 p.

[3] Chen S.T., Geller A.C., Tsao H. // Curr. Dermatol. Rep. 2013. V. 2. N 1. P. 24-34. doi 10.1007/s13671-012-0035-5

[4] Nikolaou V., Stratigos A.J. // British J. Dermatology. 2014. V. 170. N 1. P. 11-19. doi 10.1111/bjd.12492

[5] Tsao H., Olazagasti J.M., Cordoro K.M., Brewer J.D., Taylor S.C., Bordeaux J.S., Chren M.M., Sober A.J., Tegeler C., Bhushan R., Begolka W.S. // J. American Academy of Dermatology. 2015. V. 72. N 4. P. 717-723. doi 10.1016/j.jaad.2015.01.025

[6] Siegel R.L., Miller K.D., Jemal A. // CA Cancer J. Clinicians. 2016. V. 66. N 1. P. 7-30. doi $10.3322 /$ caac. 21332

[7] Siegel R.L., Miller K.D., Jemal A. // CA Cancer J. Clinicians. 2017. V. 67. N 1. P. 7-30. doi 10.3322/caac. 21387 
[8] Rahib L., Smith B.D., Aizenberg R., Rosenzweig A.B., Fleshman J.M., Matrisian L.M. // Cancer Research. 2014. V. 74. N 11. P. 2913-2921. doi 10.1158/0008-5472.CAN-14-0155

[9] Mayer J.E., Swetter S.M., Fu T., Geller A.C. // J. American Academy of Dermatology. 2014. V. 71. N 4. P. 599.e1-599.e12. doi 10.1016/j.jaad.2014.05.046

[10] Eggermont A., Spatz A., Robert C. // The Lancet. 2014. V. 1. N 383(9919). P. 816-827. doi 10.1016/S01406736(13)60802-8

[11] Demidov L.V., Sokolov D.V., Bulycheva I.V. // J. N.N. Blokhin Russian Cancer Research Center RAMS. 2007. V. 18. N 1. P. 36-41.

[12] Kittler H., Marghoob A.A., Argenziano G. // J. American Academy of Dermatology. 2016. V. 74. N 6. P. 1093-1106. doi org/10.1016/j.jaad.2015.12.038

[13] Rosendahl C., Hishon M., Cameron A., Barksdale S., Weedon D., Kittler $H$. // Dermatology Practical and Conceptual. 2014. V. 4. N 2. P. 69-75. doi 10.5826/dpc.0402a 15

[14] Deinlein T., Richtig G., Schwab C., Scarfi F., Arzberger E., Wolf I., Hofmann-Wellenhof R., Zalaudek I. // J. German Society of Dermatology. 2016. V. 14. N 2. P. 144-151. doi 10.1111/ddg.12903

[15] Gonzalez S. // Actas Dermosifiliograficas. 2009. V. 100. N 2. P. 59-69.

[16] Longo C., Ragazzi M., Ragazzi M., Nehal K., Bennassar A., Pellacani G., Guilera J.M. // Dermatologic Clinics. 2016. V. 34. N 4. P. 497-504. doi 10.1016/j.det.2016.05.012

[17] Pellacani G., De Pace B., Reggiani C., Cesinaro A.M., Argenziano G., Zalaudek I., Soyer H.P., Longo C. // Experimental Dermatology. 2014. V. 23. N 6. P. 414-418. doi 10.1111/exd.12417

[18] Müller J., Hartmann J., Bert C. // Physics in Medicine \& Biology. 2016. V. 61. N 7. P. 2646-2664. doi 10.1088/00319155/61/7/2646

[19] Petersen B., Philipsena P.A., Wulf H.C. // Photochemical \& Photobiological Sciences. 2014. V. 13. N 8. P. 1123-1125. doi $10.1039 / \mathrm{C} 4 \mathrm{PP} 00066 \mathrm{H}$

[20] Faust O., Acharya U.R., Ng E.Y.K., Hong T.J., Yu W. // Infrared Physics \& Technology. 2014. V. 66. P. 160-175. doi 10.1016/j.infrared.2014.06.001

[21] Rohrbach D.J., Muffoletto D., Huihui J., Saager $R$. Keymel K., Paquette A., Morgan J., Zeitouni N., Sunar U. // Academic Radiology. 2014. V. 21. N 2. P. 263-270. doi 10.1016/j.acra.2013.11.013

[22] Neuschmelting V., Burton N.C., Lockau H., Urich A., Harmsen S., Ntziachristos V., Kirchera M.F. // Photoacoustics. 2016. V. 4. N 1. P. 1-10. doi 10.1016/j.pacs.2015.12.001

[23] North J.P., Garrido M.C., Kolaitis N.A., LeBoit P.E. // American J. Surgical Pathology. 2014. V. 38. N 6. P. 824-831. doi 10.1097/PAS.0000000000000189

[24] Borisova E.G., Angelova L.P., Pavlova E.P. // IEEE J. Selected Topics in Quantum Electronics. 2014. V. 20. N 2. P. 211-222. doi 10.1109/JSTQE.2013.2280503

[25] Olsen J., Themstrup L., Jemec G.B. // Giornale italiano di dermatologia e venereologia. 2015. V. 150. N 5. P. 603-615.

[26] MacKinnon N., Vasefi F., Booth N., Farkas D.L. // Proc. of SPIE. 2016. V. 9711. N 1. P. 971111-971117. doi $10.1117 / 12.2222415$
[27] Zaytsev K.I., Kudrin K.G., Karasik V.E., Reshetov I.V., Yurchenko S.O. // Appl. Phys. Lett. 2015. V. 106. N 5. P. 053702. doi 10.1063/1.4907350

[28] Зайщев К.И., Черномырдин Н.В., Кудрин К.Г., Решетов И.В., Юрченко С.О. // Опт. и спектр. 2015. Т. 119. № 3. C. 404-410. doi 10.7868/S0030403415090305

[29] Lowe G.C., Saavedra A., Reed K.B., Velazquez A.I., Dronca R.S., Markovic S.N., Lohse C.M., Brewer J.D. // Mayo Clinic Proceedings. 2014. V. 89. N 1. P. 52-59. doi 10.1016/j.mayocp.2013.09.014

[30] Demidov L.V., Sokolov D.V., Bulycheva I.V. // J. N.N. Blokhin Russian Cancer Research Center RAMS. 2007. V. 18. N 1. P. $36-41$.

[31] Ganster H., Pinz P., Rohrer R., Wildling E., Binder M., Kittler H. // IEEE Transactions on Medical Imaging. 2001. V. 20. N 3. P. $233-239$. doi $10.1109 / 42.918473$

[32] Masood A., Al-Jumaily A.A. // International J. Biomedical Imaging. 2013. V. 2013. P. 1-22. doi 10.1155/2013/323268

[33] Isasi A. Gola, Zapirain García B., Méndez Zorrilla A. // Computers in Biology and Medicine. 2011. V. 41. N 9. P. 742-755. doi 10.1016/j.compbiomed.2011.06.010.

[34] Gonzalez R.C., Woods R.E. Digital Image Processing Using MATLAB. Pearson Prentice Hall, 2003. 609 p.

[35] Gonzalez R.C., Woods R.E. Digital Image Processing. Pearson Prentice Hall, 2004. 344 p.

[36] Comaniciu D., Meer P. // IEEE Transactions on Pattern Analysis and Machine Intelligence. 2002. V. 24. N 5. P. 603-619. doi 10.1109/34.1000236

[37] Comaniciu D., Meer P. // IEEE International Conference on Computer Vision. 1999. V. 2. P. 1197-1203. doi 10.1109/ICCV.1999.790416

[38] Hussain C.A., Rao D.V., Masthani S.A. // Procedia Computer Science. 2016. V. 85. P. 571-580. doi 10.1016/j.procs.2016.05.223

[39] Tuchin V.V. Tissue Optics, Light Scattering Methods and Instruments for Medical Diagnostics, Third Edition. SPIE PRESS BOOK. 2015. P. 988. doi 10.1117/3.1003040

[40] Mohammadi S., Müller H.E., Kugel H., Müller D.K., Deppe M. // Magnetic Resonance in Medicine. 2010. V. 64. N 4. P. 1047-1056. doi 10.1002/mrm.22501

[41] Bazeille S., Quidu I., Jaulin L., Malkasse J.-P. // CMM, France. 2006. P. 16-19.

[42] Rigel D.S., Russak J., Friedman R. // CA: A Cancer Journal for Clinicians. 2010. V. 60. N 5. P. 301-316. doi $10.3322 /$ caac. 20074

[43] Glazer A.M., Farberg A.S., Winkelmann R.R., Tucker N., Rigel D.S. // Dermatology Online J. 2017. V. 23. N 8. P. $13030 / \mathrm{qt} 9 \mathrm{xx} 2907 \mathrm{~s}$

[44] Kudrin K.G., Matorin O.V., Reshetov I.V. // J. Physics: Conference Series. 2015. V. 584. P. 012001. doi 10.1088/17426596/584/1/012001

[45] Samorodova O.A., Samorodov A.V. // Pattern Recognition and Image Analysis. 2016. V. 26. N 3. P. 548-551. doi 10.1134/S1054661816030020

[46] Samorodov A.V. // Pattern Recognition and Image Analysis. 2013. V. 23. N 4. P. 508-511. doi 10.1134/S1054661813040159

[47] Dobrolyubova D.A., Kravtsova T.A., Samorodova O.A., Samorodov A.V., Slavnova E.N., Volchenko N.N. // Pattern Recognition and Image Analysis. 2016. V. 26. N 3. P. 552-557. doi 10.1134/S1054661816030032 